\title{
Segmental Anomaly Leading to Wrong LeVel Disc SuRgery in CAUda Equina SYndrome
}

\author{
Gerard A. Malanga, MD, and Paul M. Cooke, MD
}

Study Design: A case report of unrecog. nized lumbarization of the $\mathrm{S}-1$ vertebral body leading to wrong-level disc surgery in a patient with acute cauda equina syndrome secondary to a large disc extrusion at the $\mathrm{L}_{4}-\mathrm{L} 5$ interspace. Laminectomy and excision were initially performed at the level of $\mathrm{L} 5-\mathrm{L} 6$.

Objectives: To review the importance of detection of lumbosacral transitional vertebrae when performing interventional procedures in the lumbar spine.

Summary of Background Data: Lumbosacral junction transitional vertebrae have been well documented. Likewise, surgery at the wrong intervertebral level has been reported as a reason for failure of lumbar spine surgery. There is also brief mention in the literature of lumbar disc surgery performed at incorrect levels in part secondary to junctional anomalies. However, there is no recorded case of wrong-level disc surgery in acute cauda equina syndrome occurring as a result of an unrecognized lumbarization or sacralization of transitional vertebrae.

Methods: A case report of wrong level disc surgery in a patient with lumbarization of the S-1 sacrum is presented. The literature, clinical course, and imaging studies are reviewed and techniques for detection of transitional vertebral bodies are discussed.

Conclusions: The presence an unde- tected congenital spinal anomaly, lumbarization of the S-1 vertebral body, resulted in mistaken intraoperative identification of the $\mathrm{L}^{-5}-5$ space in this patient resulted in decompression at the wrong level requiring a second surgery. Lumbosacral transitional vertebrae can often be overlooked on plain radiographs and magnetic resonance imaging of the lumbar spine. It is essential to be alert to the possibility of transitional vertebrae when evaluating these imaging studies, particularly in the operative treatment disc disease.

Keywords: Anomalous lumbosacral junction, herniated lumbar disc, transitional vertebra, surgery.
Lumbosacral transitional vertebrae are common congenital anomalies of the spine. Studies have found an overall prevalence ranging from $4 \%$ to $8 \%$ of the general population (1-4). The role of these vertebrae in the etiology of low back pain is controversial (5). Bertolotti (6) first described the association of back pain and sciatica with lumbosacral transitional vertebrae in 1917. In 1924, Moore (3) concluded that individuals with a sacralization had a liability and should seek an occupation requiring minimal back strain. More recent studies $(2,7)$ have found no association between lumbosacral transitional vertebrae and low back pain.

The relationship between transitional vertebrae and intervertebral disc herniation has also been described, particularly in children and adolescents $(8,9)$. In

From University of Medicine and Dentistry of New Jersey, Newark, New Jersey, and Kessler Institute for Rehabilitation, West Orange, New Jersey, Hospital for Special Surgery, New York, New York. Address Corre spondence: Gerard A. Malanga, MD, Kessler Institute for Rehabilitation, West Facility, 1199 Pleasant Valley Way, West Orange, New Jersey 07052.

E mail: gmalanga@pol.net

Funding: There was no external funding in preparation of this manuscript.

Conflict of Interst: None addition, Wigh (10) has reported lumbar disc surgery performed at incorrect levels due to nomenclature errors related to the presence of junctional anomalies. A retrospective analysis of 105 cases of failure or poor result of lumbar spine surgery concluded that surgery at the wrong level was a contributing factor (11). To our knowledge, there is no report in the literature of wrong level disc surgery in acute cauda equina syndrome as a result of a lumbosacral transitional vertebrae.

The current report is of a patient with acute cauda equina syndrome secondary to an extruded L4-5 disc that was mistakenly operated on at the L5-L6 level because of an unrecognized lumbarization of the S1 vertebral body.

\section{Case Report}

A 34-year-old male with a history of intermittent low back pain was seen in the office by his primary care physician for an exacerbation of lower back pain and spasm after a four hour car ride. He had no leg radiation and no neurological deficit. This was initially treated with rest and analgesics followed by a trial of physical therapy. The patient's symptoms failed to improve over the course of 2-3 weeks and he underwent magnetic resonance imaging (MRI) of the lumbar spine. This MRI demonstrated a central herniated nucleus pulposus at L4-5 level, small herniation at L5-L6, and the incidental finding of complete lumbarization of S1.

One week after this MRI, the patient awoke with complaints of left leg weakness and numbness of both lower extremities. He reported to the emergency room and was found to have a patchy sensory loss in L5/S1 dermatomes of both lower extremities as well as weakness of ankle dorsiflexors and everters greater on the left than the right. Plain radiographs taken in the emergency room reveal six lumbar-type vertebrae, with lumbarization of the S1 vertebral body (Fig. 1). A Foley catheter was placed and drained approximately $600 \mathrm{~mL}$ of urine.

An emergent MRI of the lumbar spine was ordered to rule out cauda equina syndrome. This demonstrated a large extruded disc at L4-5 with significant compression of the thecal sac and a protrusion at L5-S1 (Fig. 2). The patient was brought to the operating room for an emergent laminectomy. He underwent bilateral excision of herniated L4-5 disc with bilateral foraminotomies of L4- 


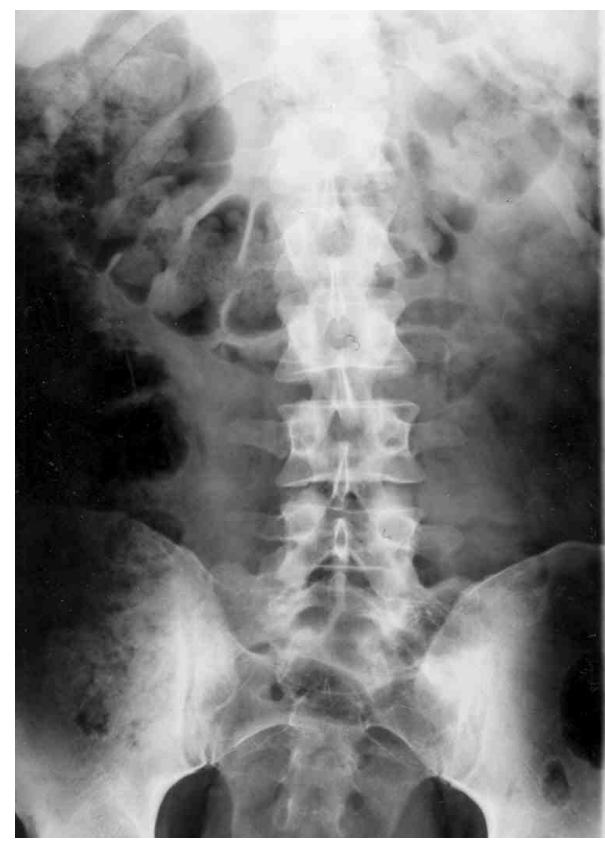

A

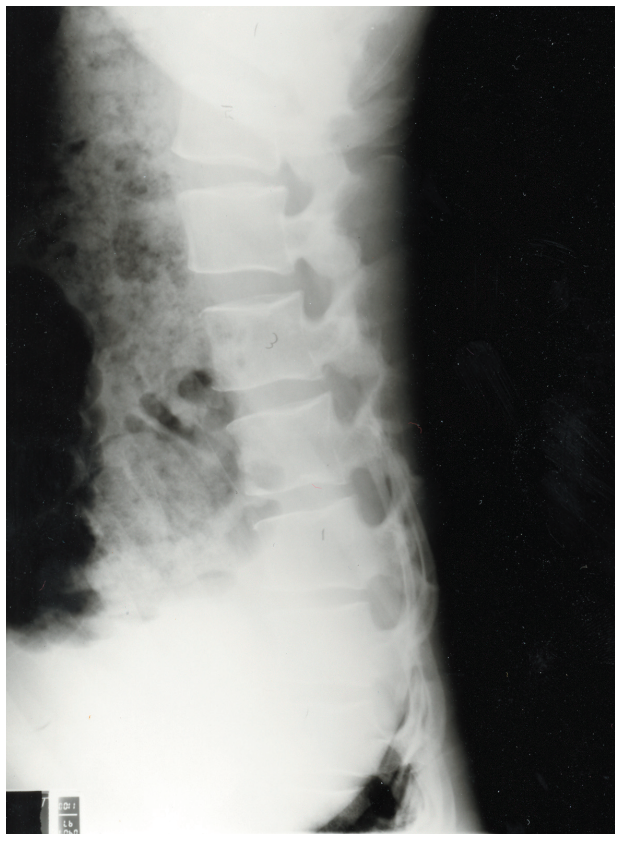

B

Fig 1. Plain radiographs of the lumbar spine (1A. AP and 1B. lateral) demonstrating six lumbar vertebrae with lumbarization of the S1 vertebral body and a functional scoliosis.

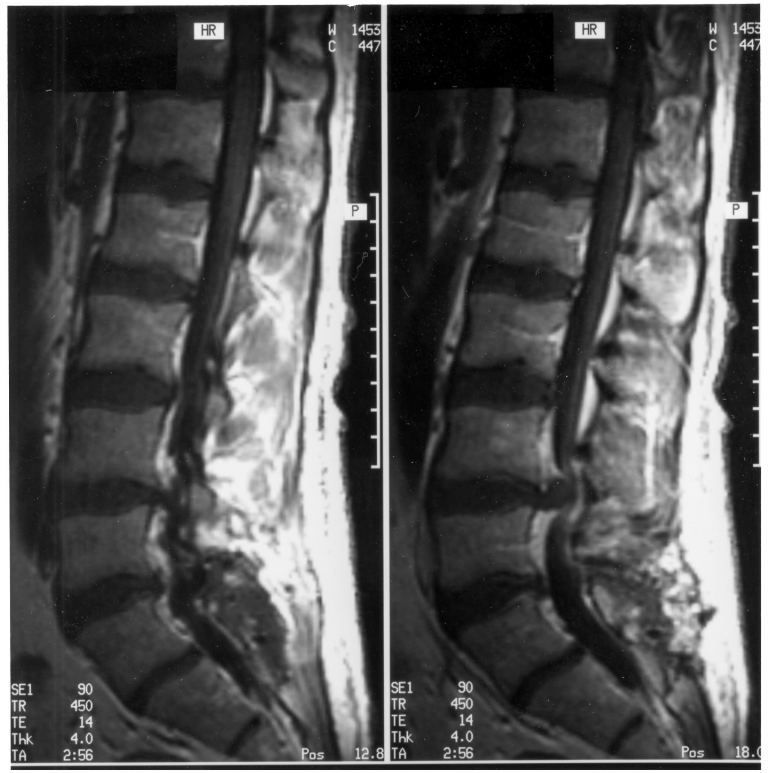

A

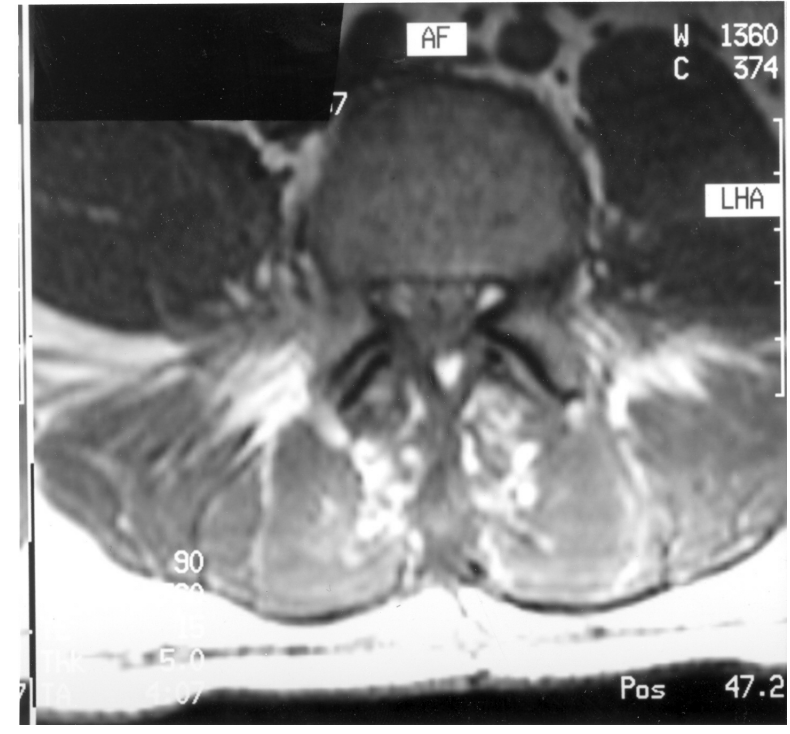

B

Fig 2. MRI of the lumbar spine with sagittal (2A and axial $2 B$ ) views demonstrating a large extruded disc at L4-5 with significant compression of the thecal sac and a protrusion at the L-5-S-1 level. Complete lumbarization of the S-1 level is also noted. 
5 and L5-S1.

Postoperatively, the patient had continued pain and profound lower extremity weakness with no active movement of his ankle plantarflexors, dorsiflexors, everters or inverters. Deep tendon reflexes were bilaterally absent at the ankle and brisk at the knee. Sensory exam revealed numbness bilaterally from the L5 to the S4-5 dermatomal regions. He had incontinent neurogenic bowel and required an indwelling Foley catheter for a neurogenic bladder.

The patient was seen for physiatric consultation and the above findings were noted. Recommendations were made regarding proper pain management as the patient was being given intramuscular Demerol for pain every 4 to 6 hours. He was mobilized out of bed to chair. Arrangements were made for transfer to a rehabilitation facility to initiate his rehabilitation as well as to reassess his neurogenic bowel and bladder and begin appropriate treatment. The patient was transferred on the following day. However, at the time of his arrival at the rehabilitation facility, he was called back to the acute care hospital after a re-reading of the intraoperative $\mathrm{x}$-ray (Fig. 3) demonstrated the operative level had been the L5-L6 rather than L4-5 interspace. This occurred due to lumbarization of the sacrum, which made the intraoperative image misleading.

The patient was again taken to the

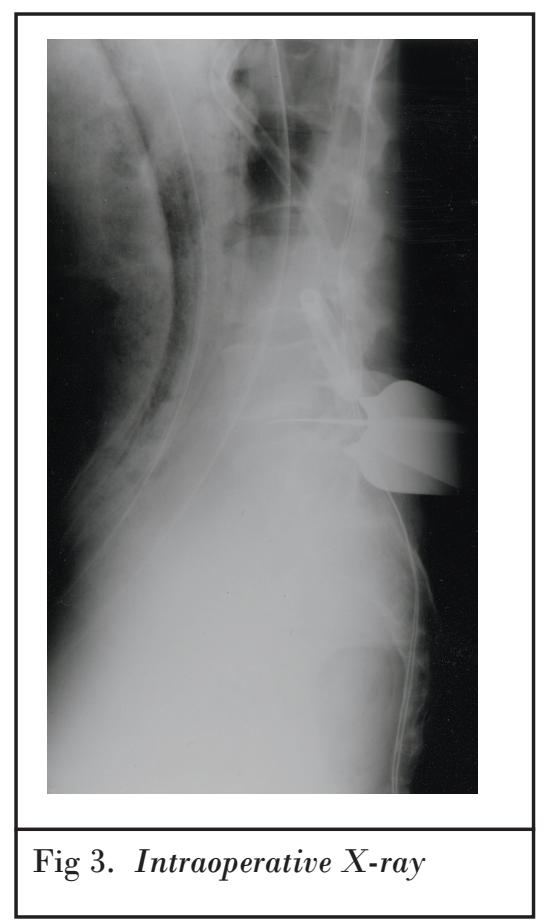

operating room and underwent decompression at the L-5 level with excision and removal of the extruded L4-5 disc fragment. This was confirmed on intraoperative localization $\mathrm{x}$-ray.

By pathology report the disc tissue specimen measured in aggregate 5.0 x $5.0 \times 4.0 \mathrm{~cm}$. The patient tolerated the procedure well. Postoperatively, he continued to be followed by a physical medicine and rehabilitation specialist and was placed on a bowel program. Physical therapy was initiated and medications for pain management were provided. He was also evaluated by urology. Urodynamic studies showed a hypotonic detrusor. He was started on Urecholine and an intermittent catheterization program. He was also placed on a tapering dose of Decadron. The patient's neurologic deficits showed gradual improvement and he was transferred back to the rehabilitation facility five days after the second surgery.

On admission to the rehabilitation facility, his lower extremity motor strength was 3-/5 for bilateral ankle dorsiflexors and extensor hallucis longus and trace for bilateral ankle plantar flexors. Sensation was intact through L4 bilaterally and was impaired from L5 through S45. Deep anal sensation was intact and voluntary anal contraction was absent. Bulbocavernosus reflex was absent.

$\mathrm{He}$ remained at the rehabilitation facility for three weeks and continued to improve. Repeat urodynamic studies revealed an atonic bladder and his bladder management on discharge was self intermittent catheterizations every 6 hours. His bowel program was discontinued as bowel function returned to normal. At the time of discharge, the patient was ambulating with bilateral forearm crutches and bilateral molded ankle foot orthoses for 500 to 1000 feet with only distant supervision. He required close supervision for stairs and distant supervision for functional transfers to the bed, car, tub and toilet. He was independent in his activities of daily living. He was discharged to home with his wife and two children.

\section{DISCUSSION}

Congenital anomalies of the spine such as lumbosacral transitional vertebrae have been well documented in the literature (1-4). A study of 4000 consecutive patients presenting for low back pain found a frequency of $6.7 \%$ of transitional vertebrae on radiographs. A control group of 1873 patients had a prevalence of $5 \%$. An increased incidence was found in families raising the possibility of a genetic component (14).

The association of low back pain and lumbosacral transitional vertebrae has been described, and is known as Bertolotti's syndrome (6). It has been postulated that transitional vertebrae may be associated with degenerative arthritis of adjacent joints and this may lead to low back pain $(1,3,6)$. However, Frymoyer et al (7) reviewed radiographs of 321 men with and without low back pain and found that those with transitional vertebrae were equally distributed. In 1997, van Tulder et al (4) performed a systematic review of published observational studies and concluded that there is no firm evidence for the presence or absence of a causal relationship between the radiographic finding of transitional vertebrae and nonspecific low back pain. However, they found few studies of acceptable methodology on this subject. Magora and Schwartz (15) found no direct relation between sacralization and low back pain in 312 subject and 148 healthy controls. They could not, however, comment on lumbarization, which had an incidence of only $0.64 \%$ in their study. A study of 46 cases of anomalous lumbosacral nerve roots found $21 \%$ to have junctional segmental spinal abnormalities as well (16).

The association between congenital spinal anomalies and lumbar disc herniation has been documented in the literature. There is a high incidence of transitional vertebrae in children and adolescents with lumbar disc herniation (8). A review of 70 operative cases of lumbar disc herniation in children and adolescents revealed twenty with congenital spinal abnormalities including spina bifida, lumbarization, and sacralization (9). In 1955, Stinchfield and Sinton (17) reported their operative experience in patients with transitional lumbosacral vertebrae. They found 30 of 31 disc herniations were at the level above the congenital anomaly. This may be the result of increased motion and stress at that segment. In 60 patients with myelographic evidence of herniated lumbar disc, there was an $83 \%$ incidence of herniated nucleus pulposus at the level cephalad to the transitional segment. This was only found in incomplete lumbarization/sacralization type of vertebrae (18). In the case we have reported, the extruded disc at L4-5 was two seg- 
ments cephalad to the transitional L6 vertebra, which is uncommon.

Failure of disc surgery immediately following the procedure has occurred due to: insufficient neural decompression, trauma to the nerve root, undiagnosed far lateral recess stenosis, unrecognized instability, persistent lateral; recess stenosis or spinal stenosis, and surgery at the wrong level (19). The actual incidence of wrong level surgery is not known but is probably extremely low. In order to order to help prevent wrong-site surgery, the North American Spine Society developed a campaign that included a checklist referred to as "sign, Mark, \& x-ray (SmaX)." Wrong disc level surgery in the case of cauda equina has never been reported and this is understandable, as the pathology in these cases is generally quite clear. In this case, the congenital anomaly of the spine and a disc protrusion at the level below the disc herniation may have been the cause for confusion and the decompression at the wrong level.

Wigh and Anthony (10) reviewed the operative reports and myelogram findings of 42 patients with transitional vertebrae and reported five cases of disc surgery performed at incorrect levels due to nomenclature error. The usual cause for this misidentification is the common practice of counting cephalad from the presumed L5 vertebra at the lumbosacral junction. Hahn (20) has proposed a method to identify transitional segments on MR imaging. Using a cervicothoracic sagittal scout MR image, the total number of vertebral bodies can be determined by counting downward from C2. Assuming seven cervical and 12 thoracic vertebrae allows for accurate detection of a lumbosacral transitional segment. It has also been suggested to identify the right renal artery on T1-weighted paramedian sagittal MR images, as this usually lies at or near the L12 disk (21). However, this technique has not been verified in published study.

The importance of identifying lumbosacral transitional vertebrae when viewing imaging studies has relevance, not only to the spine surgeon, but also to the spinal injectionist when performing diagnostic/ therapeutic spinal injection procedures. These procedures require meticulous awareness of spinal anatomy and caution particularly in patients with anomalies of the spine.

\section{ConClusion}

Wrong level disc surgery is uncommon but can result in significant consequences to the patient. One cause for wrong level surgery is congenital anomalies of the spine. These anomalies need to be recognized and carefully identified at the time of interventional procedures such as surgery. Recognition of these anomalies is also of importance to practitioners who perform spinal injections and other spinal procedures.

\section{Author Affiliation:}

Gerard A. Malanga, MD

Kessler Institute for Rehabilitation 1199 Pleasant Valley Way

West Orange, New Jersey 07052

E-mail: gmalanga@pol.net

Paul M. Cooke, MD

Hospital for Special Surgery

535 East $70^{\text {th }}$ Street

New York, NY 10021

E-mail: cookep@hss.edu

\section{RefERENCES}

1. Harmon PH. Congenital and acquired anatomic variations, including degenerative changes of the lower lumbar spine; role in production of painful back and lower extremity syndromes. Clin Orthop 1966; 44: 171-186.

2. Moore S. On the incidence of the sacralized transverse process and its significance. Radiology 1924; 2:287-301.

3. O'Connor JF, Cranley WR, McCarten KM et al. Radiographic manifestations of congenital anomalies of the spine. Radiol Clin North Am 1991; 29:407-429.

4. Van Tulder MW, Assendelft WJJ, Koes BW et al. Spinal radiographic findings and nonspecific low back pain: A systematic review of observational studies. Spine 1997; 22:427-434.

5. Esses SI, Botsford DJ. Surgical anatomy and operative approaches to the sacrum. In: Frymoyer JW (ed). The Adult Spine: Principles and Practice. $2^{\text {nd }}$ edition. Lippencott-Raven, Philadelphia, 1997:23292341.
6. Elster AD. Bertolotti's syndrome Revisited transitional vertebrae of the lumbar spine. Spine 1989;14:1373-1377.

7. Frymoyer JW, Newberg A, Pope MH et al. Spine radiographs in patients with low back pain: an epidemiological study in men. J Bone Joint Surg 1984; 66:1048-1055.

8. Epstein JA, Epstein NE, Marc J et al. Lumbar intervertebral disk herniation in teenage children: recognition and management of associated anomalies. Spine 1984; 9:427-432.

9. Kurihara A, Kataoka O. Lumbar disc herniation in children and adolescents: $A$ review of 70 operated cases and their minimum 5-year follow-up studies. Spine 1980; 5:443-451.

10. Wigh RE, Anthony HF. Transitional lumbosacral discs: probability of herniation. Spine 1981; 6:168-171.

11. Fager CA, Freidberg SR. Analysis of failures and poor results of lumbar spine surgery. Spine 1980; 5:87-94.

12. Giles RG. Vertebral anomalies. Radiology 1931; 17:1262-1266.

13. Mitchell GA. The significance of lumbosacral transitional vertebrae. Br/ Surg 1936; 24:147-158.

14. Tini PG, Wieser C, Zinn WM. The transitional vertebra of the lumbosacral spine: Its radiological classification, incidence, prevalence, and clinical significance. Rheumatol Rehabil 1977; 16:180-185.

15. Magora A, Schwartz A. Relation between the low back pain syndrome and $x$-ray findings. Scand J Rehab Med 1978; 10:135145.

16. Postacchini F, Salvatore U, Ferro L. Lumbosacral nerve root anomalies. J Bone Joint Surg 1982; 64:721-729.

17. Stinchfield FE, Sinton WA. Clinical significance of the transitional lumbosacral vertebra. JAMA 1955; 157:1107-1109.

18. Castellvi AE, Goldstein LA, Chan DP. Lumbosacral transitional vertebrae and their relationship with lumbar extradural defects. Spine 1984; 9:493-495.

19. Heher T, Dryer J, Nucci R et al. “Failed Back Surgery Syndrome" In Lefkowitz M, Lebovits A, Wlody D et al (eds). A Practical Approach to Pain Management. Lippincott Williams \& Wilkins, Philadelphia, 1996: 211-214.

20. Hahn PY, Strobel JJ, Hahn FJ. Verification of lumbosacral Segments on MR images: Identification of transitional vertebrae. $\mathrm{Ra}$ diology 1992; 182:580-581.

21. Ralston MD, Dykes TA, Applebaum BI. Verification of lumbar vertebral bodies. Radiology 1992; 185:615-616. 